\title{
A NEW ADAPTIVE METHOD FOR IMAGE COMPRESSION USING KARHUNEN-LOEVE TRANSFORM
}

Luis Torres-Urge 11

E.T.S.I. Telecomunicación 08034 Barcelona, Spain
R. Lynn Kirlin

Department of Elec. Engineering

University of Wyoming

\section{ABSTRACT}

The Karhunen-Loeve transformation (KLT) is an optimal method for encoding images in the MSE sense. only one spatially adaptive method is known to be reported in the literature. Many other suboptimal encoding methods have been developed to avoid the problems encountered in the application of the KLT. Such methods give performance which is inferior to the KLT in both MSE and visual quality, although some of them are quite efficient for first order Markov processes.

A spatial domain adaptive algorithm, which, besides giving more compression than its nonadaptive counterpart, solves the problem of ill-conditioned eigenvectors is presented. It is also shown that for a large class of images the same code set may be used, thus avoiding the computational problem normally associated with the KarhunenLoeve encoding scheme.

\section{INTRODUCTION}

The Karhunen-Loeve transform requires a large computational effort, but it is the only transform that uses the statistical properties of the image. Many researchers have used some particular models to estimate the correlation properties of the image / $1 /$. Some of these models give encouraging results and might lead us to suppose that accurate correlation modeling could be widely applied in the characterization of images for transform coding purposes. Accurate and useful modeling is possible only when the image data is of the smooth, low activity variety.

Other suboptimal transforms have been utilized to overcome the computational problem /2/. Under the assumption of first-order stationary Markov processes these transforms, specially the Discrete Cosine transform perform nearly as well as the KLT. Therefore in transform coding practice the KLT is replaced by a suitable fast unitary transform, since no fast algorithm has been reported for the Karhunen-Loeve transform.

Another approach widely used is Differential Pulse Code Modulation (DPCM). DPCM offers the tremendous advantage of its implementation facility, but on the other hand it performs closely to the KLT only for Markov processes. Not all images can be modeled as Markov processes and in fact images having even a moderate edge activity lie far from these processes.

A different problem associated with the KL scheme is the singularity of the covariance matrix. Some early experiments by Landau and Slepian describe this situation $/ 3 /$. The computational effort and the possibility of an illconditioned covariance matrix has made the KLT to be used as a bound against which the other transform coding schemes are compared.

Transform coding may be improved by adaptive methods. There are essentialiy three types of adaptation that can be made:

1. Adaptation of the transform.

2. Adaptation of bit allocation.

3. Adaptation of quantizer levels.

The aproach taken in this research is to use the basic KLT, adapting the transform to the spatially variable statistics of the image. No assumptions have been made on the class of images to be encoded. No effort is made to increase the compression through the other types of adaptation. The algorithm developed simultaneously solves the problem of singularity of the covariance matrix. Two fidelity measures are used to evaluate the results:

1. Normalized mean square error.

2. Visual evaluation. 
Only gray-scale images are discussed, however, the coding scheme is equally aplicable to three-color images, the data vectors having three times the elements.

\section{ADAPTIVE KARHUNEN-LOEVE TRANSFORMATION}

The only reported method in the literature that considers covariance matrices which adapt to the spatially variable statistics of the image is that of Tasto and wintz $/ 4 /$. Their procedure is still perhaps too complex to be practical and the classification procedures used may be imprecise. Also, it is not clear how much of the advantage of adaptation is due to the adaptive transform and how much is due to the adaptive quantization.

The block size of the transformation plays an important role in the performance of the $\mathrm{KL}$ encoding. In this work a block size of $4 \times 4$ has been chosen in order to 1) allow adaptation to the local picture characteristics and 2) compromise with computer facilities.

It is customary in the application of KLT to use zero-mean random fields. This can be achieved by finding the image mean and subtracting it from each block or by finding the mean of each block and subtracting it from each block. In each case the mean is stored and added to the final encoded image. Both alternatives have been implemented and it has been found that in the mean square error sense removing the mean of each block gives slightly better results than removing the image mean, probably because of round-off errors in processing the mean-deleted data. It can be noticed that if the mean of each block is removed and the image is then encoded with, say, two eigenvectors, the effect is the same as that of encoding the image with three eigenvectors before the image mean has been removed. All the results in this research have been obtained by first removing the mean of each block.

Formulation of the Basic Adaptive Algorithm

Let us assume we are given an $n \times n$ image and we are to encode it using the KLT. The first step is to break the image into subimages of $4 \times 4$ pixels, remove the means, and find their $16 \times 16$ autocorrelation matrix.

Depending on the amount of detail or variation among the pixels more or fewer eigenvectors will be needed to reconstruct that block to a given MSE. A question arises naturally: how many eigenvectors are needed to encode each block?. The answer, of course, is a tradeoff between estimation accuracy and the amount of compression desired.

Assume that the image is encoded using the one eigenvector corresponding to the largest eigenvalue; this eigenvector will be named the first eigenvector. Some encoded blocks will resemble almost, if not exactly, to the original blocks while others will differ considerably. A block is said to be good if the first eigenvector is sufficiently accurate to encode it, a bad block is said to be bad if more eigenvectors are needed to encode it to the desired accuracy. The approach is as follows: the original image is encoded using the first eigenvector and then the mean square errors between the originals and their corresponding encoded blocks are found. The median of all the squared errors of the blocks is then found. If any block's MSE is smaller than this value the block is said to be good; if the block's MSE is greater then the median, then the block is said to be bad, and more eigenvectors are needed. Thus we have the basis of an adaptive procedure.

The median has been chosen because it provides a robust measure of location and it is easy to find. To find the compression ratio achieved by this adaptive technique one must compute the average number of eigenvectors used in the transformation. This is given by

$\bar{n}=\sum_{i=1}^{n} i p_{i}$

where $\bar{n}$ is the number of eigenvectors and $p_{i}$ is the proportion of image for which i eigenvectors are used. The algorithm stops whenever the desired quality measure or compression has been achieved. The MSE among blocks has been chosen because is easy to find and does not require an excessive computational task.

Further Adaptability

When finding the eigenvectors of a symmetric matrix it may happen that the system is ill-conditioned. This condition arises with clustered eigenvalues. On the other hand the first eigenvalue is almost always far enough from the second, leading to a well-conditioned corresponding eigenvector. If this is not the case, a similarity transformation can be used to Eind a well-conditioned dominant eigenvalue and corresponding eigenvector $/ 5 /$. 
Assume that an image has been encoded as above using the first eigenvector and the first set of bad blocks has been found. If we subtract the encoded bad blocks from the corresponding blocks, the set of difference blocks constitutes an error image. That is, we have removed the variability corresponding to the first eigenvector and can consider the bad block error image as a new original image to be encoded. The next step is to find the autocorrelation matrix of the error image and encode it using the corresponding first eigenvector. After encoding a decision is made determining good and bad blocks. The same rule as the one used in the basic adaptive algorithm is utilized.

Summarizing the above, we list the steps as follows:

\section{Eully Adaptive Algorithm}

1. Find the mean of each block and remove it from that block.

2. Encode the mean-removed blocks using the first eigenvector of their autocorrelation matrix.

3. Find the MSE between the original and the encoded block.

4. Find the median of all the mean square errors of the blocks.

5. Label a block good or bad corresponding to whether its MSE is less than or greater than (respectively) the median of the blocks MSE's.

6. Subtract the encoded bad blocks from the corresponding original blocks and denote the resulting blocks error blocks, and denote their set the error image.

7. Find the autocorrelation matrix of the blocks in the error image.

8. Repeat steps 2 through 7 until a quality measure has been fulfilled or the desired compression has been achieved.

Each execution of steps 2 through 7 is termed and iteration.

If the eigenproblem is well-conditioned there is no need to find different autocorrelation matrices and the basic algorithm should be used. The fully adaptive algorithm takes more adaptively into account spatial variability by finding the autocorrelation matrix of the bad errors. Since it computes only the eigenvector corresponding to the largest eigenvalue, the power method or the inverse power method may be used depending on how dominant the eigenvalue is $/ 6 /$, thus speeding computations. Because it eliminates the need to find all or part of the remaining eigenvectors, computational errors and ill-conditioning are minimized.
RESULTS OF THE FULLY ADAPTIVE ALGORITHM

To check the algorithm explained above, the technique was tested with an image entitled CITY, Fig. 1. This image contains a great amount of detail with high frequency content as well as some areas of low Erequency background. The $512 \times 512$ image was divided into $4 \times 4$ blocks. The first eigenvalue of the successive autocorrelation matrices was always found to be well-conditioned.

The result of nonadaptively $\mathrm{KL}$ encoding CITY using two eigenvectors is shown in Fig. 2; and its mean square error is 0.040 . The same image encoded using the fully adaptive algorithm with one iteration, shown in Fig. 3, gives a MSE of 0.042 and no visual difference can be detected. The compression achieved in the nonadaptive case is $16 / 2=8$, and for the adaptive case it is $16 / 1,5=10.6$. This implies an increase in coding efficiency of $(10.6-8) / 8=33 \%$. We recall that the mean of each block has been previously removed and conclude that the adaptive algorithm performs at least as well as the nonadaptive one while achieving more compression. The method along with some other adaptive bit assignment scheme in the transeorm domain can achieve even more compression.

\section{VERSATILITY OF KARHUNEN-LOEVE CODE SETS}

The main drawback of the KLT is the computational effort involved in finding the covariance matrix and corresponding eigenvectors. We suggest that the Karhunen-Loeve encoding scheme has not been used at its full capability. One may wonder if there is some universal. eigenvector code set that could be employed for any kind of image. The answer at the present time is that if such a code set exists it is not yet known. However one can conjecture about the existence of this code for a particular set of images.

To validate the Eoregoing the following experiment was performed. Using the same image and block size as before, the two first eigenvectors of FACE, Fig. 4 were used to encode CITY. The striking result is shown in Fig. 5. No visual difference can be noticed when compared against Fig. 2 , and even the mean square error is the same to three significant figures. Some other images were used with the same result. This shows that for a large class of images the same set of code vectors can be used, thus avoiding the main drawback of the KL encoding scheme. If adaptive techniques are used in both the spatial and transform domain, better results are expected. 


\section{CONCLUSIONS}

Two spatially adaptive algorithms have been developed using the KarhunenLoeve transformation which give more compression than their nonadaptive counterparts. The algorithms closely parallel each other; which is applied depends on ill-conditioning and computational considerations. It is also shown that for some groups of classes of images there is no need to find the covariance matrix for each class as one code set may be efficiently applied to a variety of different image classes.

\section{ACKNOWLEDGEMENT}

We would like to thank Dr. Alireza Moghaddamjoo for his useful suggestions.

\section{REFERENCES}

/l/ Jain, A.K., "Advances in Mathematical Models for Image Processing", Proc.

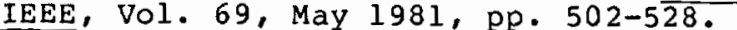

/2/ Pratt, W.K., Digital Image Processing, Wiley, New York, 1978 .

/3/ Landau, H.J. and Slepian, D., "Some Computer Experiments in Picture Processing for Bandwidth Reduction", The Bell System Technical Journal, Vol. 50 , No. 5, May-June, 1971 .

14/ Tasto, M. and Wintz, P.A. "Image Coding by Adaptive Block Quantization", IEE Transactions on Communication Technology, Vol. COM-19, No. 6, December, 1971 .

/5/ Noble, B. and Daniel, J.W., Applied Linear Algebra, Prentice Hall, 1977.

16/ Stewart, G.W.' Introduction to Matrix Computations, Academic Press, 1973.

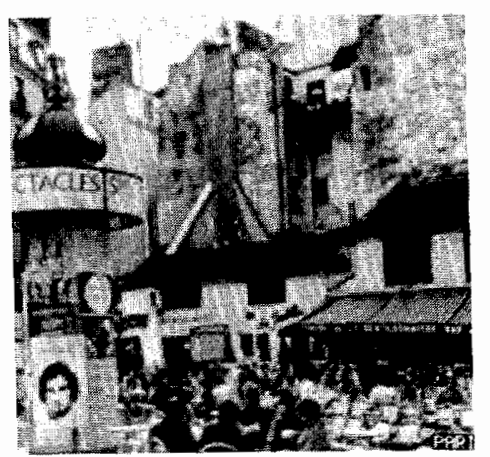

Fig. 1. Original Image of CITY.

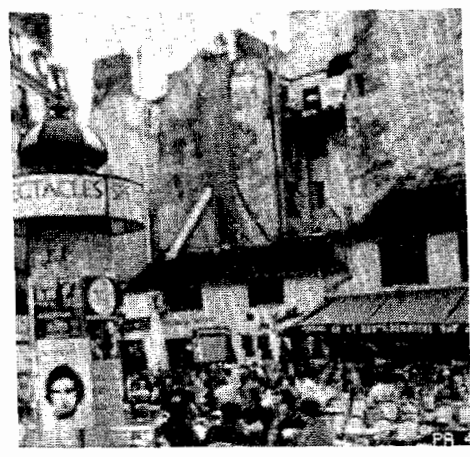

Fig. 2. Nonadaptive KL, Encoding of CITY; Two Eigenvectors.

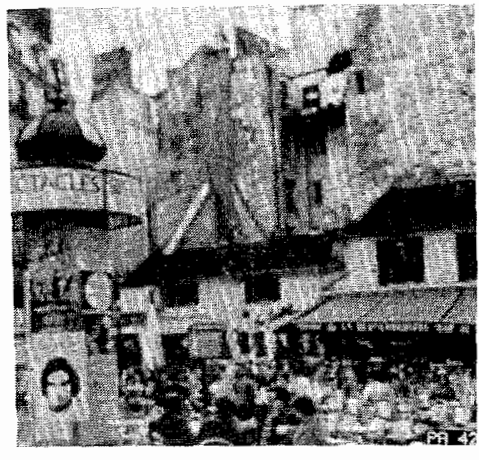

Fig. 3. Adaptive KL Encoding of CITY; One Iteration.

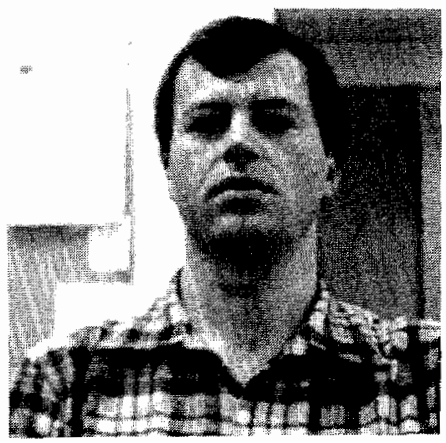

Fig. 4. Original Image of FACE.

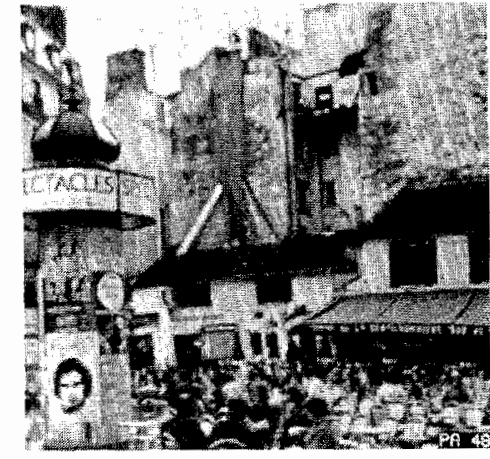

Fig. 5. CITY encoded with two eigenvectors of FACE. 